\title{
Quantum metric spaces of quantum maps
}

\author{
Maysam Maysami Sadr ${ }^{a^{*}}$ \\ ${ }^{a}$ Department of Mathematics, Institute for Advanced Studies in Basic Sciences (IASBS), Zanjan, Iran \\ *Corresponding author E-mail: sadr@iasbs.ac.ir
}

\section{Article Info}

Keywords: $C^{*}$-algebra, compact quantum metric space, Lipschitz algebra, quantum family of maps, state space 2010 AMS: 46LO5, 46L30, 54E25, $60 B 10$

Received: 21 January 2018

Accepted: 26 February 2018

Available online: 15 March 2018

\begin{abstract}
We show that any quantum family of quantum maps from a noncommutative space to a compact quantum metric space has a canonical quantum pseudo-metric structure. Here by a 'compact quantum metric space' we mean a unital $\mathrm{C}^{*}$-algebra together with a Lipschitz seminorm, in the sense of Rieffel, which induces the weak* topology on the state space of the $\mathrm{C}^{*}$-algebra. Our main result generalizes a classical result to noncommutative world.
\end{abstract}

\section{Introduction}

One of the basic ideas of Noncommutative Geometry is that any unital $\mathrm{C}^{*}$-algebra $A$ can be considered as the algebra of continuous functions on a (symbolic) compact quantum (noncommutative) space $\mathfrak{Q} A$. From this point of view, any unital *-homomorphism $\Phi: B \rightarrow A$ between unital $\mathrm{C}^{*}$-algebras can be interpreted as a quantum map $\mathfrak{Q} \Phi$ from $\mathfrak{Q} A$ into $\mathfrak{Q} B$. There are many notions in Topology and Geometry that can be translate into NC language. The notion of quantum family of (quantum) maps, defined by Woronowicz [16] and Sołtan [15] (see also $[10,11,12])$, conclude from the following fact: "Every map $f$ from $X$ to the set of all maps from $Y$ to $Z$ (or in other word, any family of maps from $Y$ to $Z$ parameterized by $f$ with parameters $x$ in $X)$ can be considered as a map $\tilde{f}: X \times Y \rightarrow Z$ defined by $\tilde{f}(x, y)=f(x)(y)$." A translation of this to noncommutative language is as follows.

Definition 1.1. ([10, 11, 12, 15, 16] Let B,C be unital $C^{*}$-algebras. A quantum family of morphisms from B to $C$ (or, a quantum family of maps from $\mathfrak{Q} C$ to $\mathfrak{Q} B)$ is a pair $(A, \Phi)$ consisting of a unital $C^{*}$-algebra $A$ and a unital $*$-homomorphism $\Phi: B \rightarrow C \otimes A$, where $\otimes$ denotes the spatial tensor product of $C^{*}$-algebras.

Another concept that can be translate from Geometry into NC Geometry, is distance or metric. Marc Rieffel, by using the notion of order unite spaces, has developed the notion of quantum metric space in a series of papers [5, 6, 7, 8, 9]. For two other different notions of quantum metric see $[3,13,14]$. Here, we deals with special examples of Rieffel's quantum metric spaces, stated in the $C^{*}$-algebraic formalism. The aim of this note is to show that any quantum family of maps from a quantum space to a compact quantum metric space has a canonical quantum pseudo-metric structure. We are motivated by the following trivial fact: Let $(Z, d)$ be a metric space and $f: X \times Y \rightarrow Z$ be a family of maps from $Y$ to $Z$, then $X$ has a pseudo-metric $\rho$ defined by

$$
\rho\left(x, x^{\prime}\right)=\sup _{y \in Y} d\left(f(x, y), f\left(x^{\prime}, y\right)\right) .
$$

In Section 2 we introduce the notion of compact quantum pseudo-metric space. In Section 3 we define a natural compact quantum pseudo-metric space structure on any quantum family of maps from a quantum space to a compact quantum metric space. In Section 4 we examine our definition in the classical case.

\section{Compact quantum pseudo-metric spaces}

By a pseudo-metric $d$ on a set $X$ we mean a positive valued function on $X \times X$ which is symmetric, satisfies triangle inequality, and $d(x, x)=0$ for every $x \in X$. For any topological space $X$ with topology $\tau$ (resp. pseudo-metric space $(X, d)) \mathbf{C}(X, \tau)($ resp. $\mathbf{C}(X, d))$ denotes the 
$\mathrm{C}^{*}$-algebra of all continuous bounded complex valued maps on $X$ with the uniform norm. For a pseudo-metric $d$, $\tau_{d}$ denotes the topology induced by $d$. Let $(X, d)$ be a pseudo-metric space. For every $f \in \mathbf{C}(X, d)$, the Lipschitz semi norm $\|f\|_{d}$ is defined by

$$
\|f\|_{d}=\sup \left\{\frac{\left|f(x)-f\left(x^{\prime}\right)\right|}{d\left(x, x^{\prime}\right)}: x, x^{\prime} \in X, d\left(x, x^{\prime}\right) \neq 0\right\} .
$$

Also, the Lipschitz algebra of $(X, d)$ is defined by,

$$
\mathbf{L i p}(X, d)=\left\{f \in \mathbf{C}(X, d):\|f\|_{d}<\infty\right\} .
$$

We need the following simple lemma.

Lemma 2.1. Let $(X, d)$ be a pseudo-metric space and a be a complex valued map on $X$. Then $a \in \operatorname{Lip}(X, d)$ and $\|a\|_{d} \leq 1$ if and only if $\left|a(x)-a\left(x^{\prime}\right)\right| \leq d\left(x, x^{\prime}\right)$ for every $x, x^{\prime} \in X$. In particular, if $b \in \mathbf{C}(X, d)$, then $\|b\|_{d}=0$ if and only if $b$ is a constant map.

Proof. Let $a \in \operatorname{Lip}(X, d)$ and $\|a\|_{d} \leq 1$. Suppose that $x, x^{\prime} \in X$. If $d\left(x, x^{\prime}\right)=0$, then $a(x)=a\left(x^{\prime}\right)$, since $a$ is continuous with $\tau_{d}$. If $d\left(x, x^{\prime}\right) \neq 0$, then $1 \geq\|a\|_{d} \geq \frac{\left|a(x)-a\left(x^{\prime}\right)\right|}{d\left(x, x^{\prime}\right)}$, and thus $\left|a(x)-a\left(x^{\prime}\right)\right| \leq d\left(x, x^{\prime}\right)$. The other direction is trivial.

For any $C^{*}$-algebra $\mathfrak{A}, S(\mathfrak{A})$ denotes the state space of $\mathfrak{A}$ with w* topology. If $\mathfrak{A}$ is unital, $1_{\mathfrak{A}}$ denotes the unit element of $\mathfrak{A}$.

Let $\mathscr{A}$ be a self adjoint linear subspace of the $\mathrm{C}^{*}$-algebra $\mathfrak{A}$, and let $L: \mathscr{A} \rightarrow[0, \infty)$ be a semi norm on $\mathscr{A}$. Connes has pointed out [1], [2], that one can define a pseudo-metric $\rho_{L}$ on $S(\mathfrak{A})$ by

$$
\rho_{L}(\mu, v)=\sup \{|\mu(a)-v(a)|: a \in \mathscr{A}, L(a) \leq 1\} \quad(\mu, v \in S(\mathfrak{A})) .
$$

Note that $\rho_{L}$ can take values $+\infty$ and 0 for different states of $\mathfrak{A}$. Conversely, let $d$ be a pseudo-metric on $S(A)$ (such that the topology induced by $d$ on $S(\mathfrak{A})$ is not necessarily w* topology). Define a semi norm $L_{d}: \mathfrak{A} \rightarrow[0,+\infty]$ by

$$
L_{d}(a)=\sup \left\{\frac{|\mu(a)-v(a)|}{d(\mu, v)}: \mu, v \in S(\mathfrak{A}), d(\mu, v) \neq 0\right\} \quad(a \in \mathfrak{A}) .
$$

Note that $L_{d}(a)=L_{d}\left(a^{*}\right)$ for every $a \in \mathfrak{A}$.

Let $(X, d)$ be a compact metric space. Consider the Lipschitz semi norm

$$
\|\cdot\|_{d}: \mathbf{L i p}(X, d) \subset \mathbf{C}(X, d) \rightarrow[0,+\infty) .
$$

Then it is easily checked that the semi norm $\rho_{\|\cdot\|_{d}}$ on the state space of $\mathbf{C}(X, d)$ is a metric, called Monge-Kantorovich metric [4]. It is well known that the topology induced by $\rho_{\|\cdot\|_{d}}$, is the w* topology, and for every $x, y \in X, d(x, y)=\rho_{\|\cdot\|_{d}}\left(\delta_{x}, \delta_{y}\right)$, where $\delta: X \rightarrow \mathbf{C}(X, d)^{*}$ is the point mass measure map.

Proposition 2.2. Let $(X, \tau)$ be a compact Hausdorff space and $d$ be a pseudo-metric on $X$ such that the topology induced by $d$ on $X$ is weaker than $\tau$, i.e. $\tau_{d} \subset \tau$. Consider the Lipschitz semi norm $\|\cdot\|_{d}: \operatorname{Lip}(X, d) \subset \mathbf{C}(X, \tau) \rightarrow[0,+\infty)$ and let $\rho=\rho_{\|\cdot\|_{d}}$. Then the following are satisfied.

i) $d(x, y)=\rho\left(\delta_{x}, \delta_{y}\right)$, for every $x, y \in X$.

ii) $L_{\rho}=\|\cdot\|_{d}$ on $\mathbf{C}(X, d) \subset \mathbf{C}(X, \tau)$.

iii) Let $a \in \mathbf{C}(X, \tau)$, then $a \in \mathbf{C}(X, d)$ if and only if the map $v \longmapsto v(a)$ on $S(\mathbf{C}(X, \tau))$ is continuous with $\rho$.

iv) the topology induced by $\rho$ on $S(\mathbf{C}(X, \tau))$ is weaker than the $w^{*}$ topology.

Proof. i) Let $x, y$ be in $X$. Suppose that $a \in \operatorname{Lip}(X, d)$ and $\|a\|_{d} \leq 1$. Then by Lemma $2.1,\left|\delta_{x}(a)-\delta_{y}(a)\right|=|a(x)-a(y)| \leq d(x, y)$, and thus by definition of $\rho$, we have $\rho\left(\delta_{x}, \delta_{y}\right) \leq d(x, y)$. Conversely, let $a_{x} \in \mathbf{C}(X, d)$ be defined by $a_{x}(z)=d(x, z)(z \in X)$; then for every $x^{\prime}, y^{\prime} \in X$, $\left|a_{x}\left(x^{\prime}\right)-a_{x}\left(y^{\prime}\right)\right|=\left|d\left(x, x^{\prime}\right)-d\left(x, y^{\prime}\right)\right| \leq d\left(x^{\prime}, y^{\prime}\right)$, and thus by lemma 2.1, $a \in \mathbf{L i p}(X, d)$ and $\|a\|_{d} \leq 1$. Now, we have

$$
\rho\left(\delta_{x}, \delta_{y}\right) \geq\left|\delta_{x}\left(a_{x}\right)-\delta_{y}\left(a_{x}\right)\right|=\left|a_{x}(x)-a_{x}(y)\right|=d(x, y) .
$$

ii) By i) and definitions of $L_{\rho}$ and $\|\cdot\|_{d}$, it is clear that $\|\cdot\|_{d} \leq L_{\rho}$ on $\mathbf{C}(X, \tau)$.

Let $a \in \mathbf{C}(X, d)$. If $\|a\|_{d}=0$, then by Lemma 2.1, $a$ is a constant map and thus $L_{\rho}(a)=0$. If $\|a\|_{d}=\infty$ then $L_{\rho}(a)=\infty$ since $\|a\|_{d} \leq L_{\rho}(a)$. Thus suppose that $0<\|a\|<\infty$. Then for every $\mu, v \in S(\mathbf{C}(X, \tau))$, we have

$$
\rho(\mu, v) \geq\left|\mu\left(\frac{a}{\|a\|_{d}}\right)-v\left(\frac{a}{\|a\|_{d}}\right)\right|=\frac{|\mu(a)-v(a)|}{\|a\|_{d}}
$$

and thus if $\rho(\mu, v) \neq 0$ then $\|a\|_{d} \geq \frac{|\mu(a)-v(a)|}{\rho(\mu, v)}$. Therefore,

$$
\|a\|_{d} \geq \sup \left\{\frac{|\mu(a)-v(a)|}{\rho(\mu, v)}: \quad \mu, v \in S(\mathbf{C}(X, \tau)), \rho(\mu, v) \neq 0\right\}=L_{\rho}(a) .
$$

iii) The 'if' part is an immediate consequence of i). For the other direction, we need some notations: Let $\sim$ be the equivalence relation on $X$ defined by $x \sim x^{\prime} \Leftrightarrow d\left(x, x^{\prime}\right)=0$. Let $Y=X / \sim$ and let ${ }^{\wedge}: X \rightarrow Y$ be the canonical projection. Then $\hat{d}$, defined by $\hat{d}\left(\hat{x}_{1}, \hat{x}_{2}\right)=d\left(x_{1}, x_{2}\right)$, is a well defined metric on $Y$, and ${ }^{\wedge}$ is an isometry between $(X, d)$ and $(Y, \hat{d})$. Thus the $\mathrm{C}^{*}$-algebras $\mathbf{C}(X, d)$ and $\mathbf{C}(Y, \hat{d})$, and the Lipschitz algebras $\left(\operatorname{Lip}(X, d),\|\cdot\|_{d}\right)$ and $\left(\operatorname{Lip}(Y, \hat{d}),\|\cdot\|_{\hat{d}}\right)$ are isometric isomorph. In particular, the topology induced by $\rho$ on $S(\mathbf{C}(X, d))$ is the w* topology, since as mentioned above the Monge-Kantorovich metric $\rho_{\|\cdot\|_{\hat{d}}}$ induces the w* topology on $S(\mathbf{C}(Y, \hat{d}))$. Consider the canonical embedding $\Phi: \mathbf{C}(X, d) \rightarrow \mathbf{C}(X, \tau)$. For every $v, v^{\prime} \in S(\mathbf{C}(X, \tau)), v \circ \Phi$ and $v^{\prime} \circ \Phi$ are in $S(\mathbf{C}(X, d))$ and

$$
\rho\left(v, v^{\prime}\right)=\rho\left(v \circ \Phi, v^{\prime} \circ \Phi\right) \text {. }
$$


Now, let $a \in \mathbf{C}(X, d)$ and $v_{i} \rightarrow v$ be a convergent net in $S(\mathbf{C}(X, \tau))$ with $\rho$. Then $v_{i} \circ \Phi \rightarrow v \circ \Phi$ is a convergent net in $S(\mathbf{C}(X, d))$ with $\rho$, and since the topology induced by $\rho$ agrees with the w* topology on $S(\mathbf{C}(X, d))$, we have

$$
v_{i}(a)=v_{i} \circ \Phi(a) \rightarrow v \circ \Phi(a)=v(a) .
$$

Thus we get the desired result.

iv) Let $v_{i} \rightarrow v$ be a convergent net in $S(\mathbf{C}(X, \tau))$ with w* topology. Thus as in the proof of iii), $v_{i} \circ \Phi \rightarrow v \circ \Phi$ with $\rho$, and by (2.2), $v_{i} \rightarrow v$ in $S(\mathbf{C}(X, \tau))$ with the topology induced by $\rho$. This completes the proof of iv).

Definition 2.3. By a compact quantum pseudo-metric space (QSM space, for short) we mean a triple ( $\mathfrak{A}, \mathscr{A}, L)$, where $\mathfrak{A}$ is a unital $C^{*}$-algebra, $\mathscr{A}$ is a self adjoint linear subspace of $\mathfrak{A}$ with $1_{\mathfrak{A}} \in \mathscr{A}$, and $L: \mathscr{A} \rightarrow[0,+\infty)$ is a semi norm such that

(a) $L(a)=L\left(a^{*}\right)$ for every $a \in \mathscr{A}$,

(b) for every $a \in \mathscr{A}, L(a)=0$ if and only if $a \in \mathbb{C} 1_{\mathfrak{A}}$, and

(c) the topology induced by the pseudo-metric $\rho_{L}$ on $S(\mathfrak{A})$ is weaker than the $w^{*}$ topology.

As an immediate corollary of the definition, for any compact quantum pseudo-metric space $(\mathfrak{A}, \mathscr{A}, L)$, the topology induced by $\rho_{L}$ on $S(\mathfrak{A})$ is compact and in particular the diameter of $S(\mathfrak{A})$ under $\rho_{L}$ is finite.

Proposition 2.4. Let $(\mathfrak{A}, \mathscr{A}, L)$ be a $Q S M$ space. Then, for every $a \in \mathscr{A}$, the map $\mu \longmapsto \mu(a)$ on $S(\mathfrak{A})$ is continuous with topology induced by $\rho_{L}$.

Proof. Straightforward.

Definition 2.5. A $Q S M$ space $(\mathfrak{A}, \mathscr{A}, L)$ is called a compact quantum metric space (QM space, for short) if $\mathscr{A}$ is a dense subspace of $\mathfrak{A}$.

Let $(\mathfrak{A}, \mathscr{A}, L)$ be a $\mathrm{QM}$ space and $\mu, v$ be two different states of $\mathfrak{A}$. Then since $\mathscr{A}$ is dense in $\mathfrak{A}$, there is $a \in \mathscr{A}$ such that $\mu(a) \neq v(a)$. Thus (by (2.1)) $\rho_{L}$ is a metric on $S(\mathfrak{A})$. It is an elementary result in Topology that any Hausdorff topology $\tau$ weaker than a compact Hausdorrf topology $\tau^{\prime}$ on a set $X$, is equal to the same topology $\tau^{\prime}$. Using this, we conclude that the topology induced by $\rho_{L}$ on $S(\mathfrak{A})$ is the w* topology.

Example 2.6. Let $(X, d)$ be a compact metric space. Then

$$
\left(\mathbf{C}(X, d), \mathbf{L i p}(X, d),\|\cdot\|_{d}\right)
$$

is a compact quantum metric space.

Example 2.7. Let $(X, \tau)$ be a compact Hausdorff space and let $d$ be a pseudo-metric on $X$ such that $\tau_{d} \subset \tau$. Then Proposition 2.2 and Lemma 2.1, show

$$
\left(\mathbf{C}(X, \tau), \mathbf{L i p}(X, d),\|\cdot\|_{d}\right)
$$

is a compact quantum pseudo-metric space.

Remark 2.8. Let $(\mathfrak{A}, \mathscr{A}, L)$ be a $Q M$ space and $A \subset \mathscr{A}$ be the linear subspace of all self-adjoint elements of $\mathscr{A}$. Then $A$ is an order unite space and $\left(A,\left.L\right|_{A}\right)$ is a compact quantum metric space in the sense of Rieffel's definition [7].

Lemma 2.9. Let $\mathfrak{A}$ be a $C^{*}$-algebra with the $C^{*}$-norm $\|\cdot\|, \mathscr{A}$ be a self adjoint linear subspace of $\mathfrak{A}$ containing $1_{\mathfrak{A}}$ and $L: \mathscr{A} \rightarrow[0,+\infty)$ be a semi norm such that for every $a \in \mathscr{A}, L(a)=0$ if and only if $a \in \mathbb{C} 1_{\mathfrak{A}}$. Let $\tilde{L}$ and $\|\cdot\|$ denote the quotient norm of $L$ and $\|\cdot\|$ on $\frac{\mathscr{A}}{\mathbb{C} 1_{\mathfrak{A}}}$ and $\frac{\mathfrak{A}}{\mathbb{C} \mathfrak{A}_{\mathfrak{A}}}$, respectively. Suppose that the image of $\{a \in \mathscr{A}: L(a) \leq 1\}$ in $\frac{\mathfrak{A}}{\mathbb{C 1}_{\mathfrak{A}}}$ is totally bounded for $\|\cdot\|$. Then the topology induced by $\rho_{L}$ on $S(\mathfrak{A})$ is weaker than the $w^{*}$ topology.

Proof. See Theorem 1.8 of [5].

Example 2.10. Let $\mathfrak{A}$ be a finite dimensional $C^{*}$-algebra and $N$ be a Banach space norm on $\mathfrak{A}$ such that $N(a)=N\left(a^{*}\right)$ for every a $\in \mathfrak{A}$. Let the semi norm $N_{0}: \mathfrak{A} \rightarrow[0, \infty)$ be defined by

$$
N_{0}=\inf \left\{N\left(a+\lambda 1_{\mathfrak{A}}\right): \quad \lambda \in \mathbb{C}\right\} .
$$

Since $\mathfrak{A}$ is finite dimensional, the $C^{*}$-norm of $\mathfrak{A}$ and $N$ are equivalent. Thus the image $K$ of $\left\{a \in \mathfrak{A}: N_{0}(a) \leq 1\right\}$ is closed and bounded in $\frac{\mathfrak{A}}{\mathbb{C} 1_{\mathfrak{A}}}$. Again, since $\mathfrak{A}$ is finite dimensional, $K$ is compact and thus totally bounded for the quotient norm of the $C^{*}-$ norm. Thus by Lemma 2.9 , $\left(\mathfrak{A}, \mathfrak{A}, N_{0}\right)$ is a $Q M$ space.

Example 2.11. Let $G$ be a compact Hausdorff group with identity element e. Let $\ell$ be a length function on $G$, i.e. $\ell$ is a continuous non negative real valued function on $G$ such that

(i) $\ell\left(g g^{\prime}\right) \leq \ell(g)+\ell\left(g^{\prime}\right)$, for every $g, g^{\prime} \in G$,

(ii) $\ell(g)=\ell\left(g^{-1}\right)$ for every $g \in G$, and

(iii) $\ell(g)=0$ if and only if $g=e$.

Let $\mathfrak{A}$ be a unital $C^{*}$-algebra with a strongly continuous action $\cdot: G \times \mathfrak{A} \rightarrow \mathfrak{A}$ of $G$ by automorphisms of $\mathfrak{A}$, i.e.

(a) for every $g \in G$ the map $a \longmapsto g \cdot a$ is $a *$-automorphism of $\mathfrak{A}$,

(b) $e \cdot a=$ a for every $a \in \mathfrak{A}$,

(c) $g \cdot\left(g^{\prime} \cdot a\right)=\left(g g^{\prime}\right) \cdot a$, for every $g, g^{\prime} \in G, a \in A$, and

(d) if $g_{i} \rightarrow g$ is a convergent net in $G$ and $a \in \mathfrak{A}$, then $g_{i} \cdot a \rightarrow g \cdot a$ with the $C^{*}$-norm of $\mathfrak{A}$. 
Define a semi norm L on $\mathfrak{A}$ by

$$
L(a)=\sup \left\{\frac{\|g \cdot a-a\|}{\ell(g)}: \quad g \in G, g \neq e\right\} \quad(a \in \mathfrak{A}) .
$$

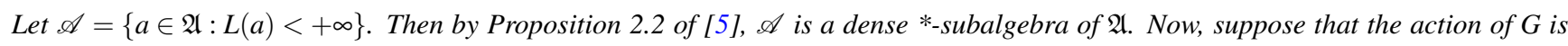
ergodic, i.e. if $a \in \mathfrak{A}$ and for every $g \in G, g \cdot a=a$, then $a \in \mathbb{C} 1_{\mathfrak{A}}$. Then it is trivial that $L(a)=0$ if and only if $a \in \mathbb{C} 1_{\mathfrak{A}}$. Rieffel has proved [5, Theorem 2.3], that the topology induced by $\rho_{L}$ on $S(\mathfrak{A})$ agrees with the $w^{*}$ topology. Thus $(\mathfrak{A}, \mathscr{A}, L)$ is a $Q M$ space.

For some other examples that completely match our notion of QM space, see [5]. As we will see in the next section, using quantum family of morphisms we can construct many QSM spaces from a QSM space.

\section{The main definition}

We need the following simple topological lemma.

Lemma 3.1. Let $Y$ be a compact space, $X$ be an arbitrary space and $(Z, \rho)$ be a pseudo-metric space. Also, let $\mathbf{C}(Y, Z)$ be the space of all continuous maps from $Y$ to $Z$, with the pseudo-metric $\hat{\rho}$ defined by

$$
\hat{\rho}(f, g)=\sup \{\rho(f(y), g(y)): \quad y \in Y\} \quad(f, g \in \mathbf{C}(Y, Z))
$$

Suppose that $F: Y \times X \rightarrow Z$ is a continuous map. Then the map $\tilde{F}: X \rightarrow \mathbf{C}(Y, Z)$, defined by $\tilde{F}(x)(y)=F(y, x)$ is continuous.

Proof. Let $x_{0} \in X$ and $\varepsilon>0$ be arbitrary. Since $F$ is continuous, for every $y \in Y$, there are open sets $U_{y}, V_{y}$ in $X$ and $Y$ respectively, such that $\left(y, x_{0}\right) \in V_{y} \times U_{y}$ and $\rho\left(F\left(y, x_{0}\right), F\left(y^{\prime}, x\right)\right)<\varepsilon / 2$ for every $\left(y^{\prime}, x\right) \in V_{y} \times U_{y}$. Since $Y$ is compact, there are $y_{1}, \cdots, y_{n} \in Y$ such that $Y=\cup_{i=1}^{n} V_{y_{i}}$. Let $W$ be the open set $\cap_{i=1}^{n} U_{y_{i}}$. Let $x \in W$ and $y \in Y$ be arbitrary. Then for some $i(i=1, \cdots, n), y$ belongs to $V_{y_{i}}$ and we have,

$$
\rho\left(F(y, x), F\left(y, x_{0}\right)\right) \leq \rho\left(F(y, x), F\left(y_{i}, x_{0}\right)\right)+\rho\left(F\left(y_{i}, x_{0}\right), F\left(y, x_{0}\right)\right)<\varepsilon
$$

Thus we have $\hat{\rho}\left(\tilde{F}(x), \tilde{F}\left(x_{0}\right)\right)<\varepsilon$ for every $x \in W$. The proof is complete.

Let $(\mathfrak{A}, \mathscr{A}, L)$ be a QSM space, $\mathfrak{B}$ be a unital $C^{*}$-algebra, and $(\mathfrak{C}, \Phi)$ be a quantum family of morphisms from $\mathfrak{A}$ to $\mathfrak{B}, \Phi: \mathfrak{A} \rightarrow \mathfrak{B} \otimes \mathfrak{C}$. Let $d$ be a pseudo-metric on $S(\mathfrak{C})$, defined by

$$
d\left(v, v^{\prime}\right)=\sup \left\{\rho_{L}\left((\mu \otimes v) \Phi,\left(\mu \otimes v^{\prime}\right) \Phi\right): \mu \in S(\mathfrak{B})\right\} \quad\left(v, v^{\prime} \in S(\mathfrak{C})\right) .
$$

Proposition 3.2. With the above assumptions, let $\mathscr{C}$ be the linear space of all $c \in \mathfrak{C}$ such that the map $v \longmapsto v(c)$ on $S(\mathfrak{C})$ is continuous with the topology induced by $d$, and $L_{d}(c)<\infty$. Then the following are satisfied.

i) $\mathscr{C}$ is a self adjoint linear subspace of $\mathfrak{C}$ and $1_{\mathfrak{C}} \in \mathscr{C}$.

ii) For every $c \in \mathscr{C}, L_{d}(c)=0$ if and only if $c \in \mathbb{C} 1_{\mathfrak{C}}$.

iii) The topology induced by $d$ on $S(\mathfrak{C})$ is weaker than the $w^{*}$ topology.

iv) With the restriction of the domain of $L_{d}$ to $\mathscr{C}, \rho_{L_{d}} \leq d$.

v) The topology induced by $\rho_{L_{d}}$ on $S(\mathfrak{C})$ is weaker than the $w^{*}$ topology.

Proof. i) is easily checked.

ii) Let $c$ be in $\mathscr{C}$ and $L_{d}(c)=0$. By Lemma 2.1, the map $v \longmapsto v(c)$ on $S(\mathfrak{C})$ is constant, and thus $c \in \mathbb{C} 1_{\mathfrak{C}}$.

iii) Apply Lemma 3.1, with $X=S(\mathfrak{C}), Y=S(\mathfrak{B}), Z=S(\mathfrak{A}), \rho=\rho_{L}$ and $F: Y \times X \rightarrow Z$ defined by

$$
F(\mu, v)=(\mu \otimes v) \Phi \quad(\mu \in Y, v \in X) .
$$

We get $\tilde{F}: X \rightarrow \mathbf{C}(Y, Z)$ is continuous with the metric $\hat{\rho}$ on $\mathbf{C}(Y, Z)$. On the other hand, for every $v, v^{\prime}$ we have $d\left(v, v^{\prime}\right)=\hat{\rho}\left(\tilde{F}(v), \tilde{F}\left(v^{\prime}\right)\right)$. Thus, if $v_{i} \rightarrow v$ is a convergent net in $X$ with $\mathrm{w}^{*}$ topology, then

$$
d\left(v_{i}, v\right)=\hat{\rho}\left(\tilde{F}\left(v_{i}\right), \tilde{F}(v)\right) \rightarrow 0 .
$$

This implies that the topology induced by $d$ is weaker than the $\mathrm{w}^{*}$ topology.

iv) Let $v, v^{\prime}$ be in $S(\mathfrak{C})$. If $d\left(v, v^{\prime}\right)=0$ then for every $c \in \mathscr{C}, v(c)=v^{\prime}(c)$ (since the map $\mu \longmapsto \mu(c)$ is continuous with $d$ ) and thus by the definition of $\rho_{L_{d}}, \rho_{L_{d}}\left(v, v^{\prime}\right)=0$. Thus suppose that $d\left(v, v^{\prime}\right) \neq 0$. Let $c \in \mathscr{C}$ with $L_{d}(c) \leq 1$. Then $1 \geq L_{d}(c) \geq \frac{\left|v(c)-v^{\prime}(c)\right|}{d\left(v, v^{\prime}\right)}$, and thus $\left|v(c)-v^{\prime}(c)\right| \leq d\left(v, v^{\prime}\right)$. Therefore

$$
\rho_{L_{d}}\left(v, v^{\prime}\right) \leq d\left(v, v^{\prime}\right)
$$

v) follows directly from iv) and iii).

Definition 3.3. With the above assumptions, Proposition 3.2, shows that $\left(\mathfrak{C}, \mathscr{C}, L_{d}\right)$ is a QSM space that is called QSM space induced by the QSM space $(\mathfrak{A}, \mathscr{A}, L)$ and quantum family of maps $(\mathfrak{C}, \Phi)$.

Lemma 3.4. With the above assumptions, let $a \in \mathscr{A}$ and let $\mu \in S(\mathfrak{B})$. Then $c=\left(\mu \otimes i d_{\mathfrak{C}}\right) \Phi(a)$ is in $\mathscr{C}$, and $L_{d}(c) \leq L(a)$. 
Proof. We first show that $L_{d}(c) \leq L(a)(<\infty)$. If $L(a)=0$ then $a \in \mathbb{C} 1_{\mathfrak{A}}$ and thus $c \in \mathbb{C} 1_{\mathfrak{C}}$ and $L_{d}(c)=0$. Suppose that $L(a) \neq 0$. We prove that for every $v, v^{\prime} \in S(\mathfrak{C})$ with $\bar{d}\left(v, v^{\prime}\right) \neq 0$,

$$
\frac{\left|v(c)-v^{\prime}(c)\right|}{d\left(v, v^{\prime}\right)} \leq L(a)
$$

Let $v, v^{\prime} \in S(\mathfrak{C})$ be such that $d\left(v, v^{\prime}\right) \neq 0$. If $\left|v(c)-v^{\prime}(c)\right|=0$, then (3.1) is satisfied. Suppose that

$$
\left|v(c)-v^{\prime}(c)\right|=\left|(\mu \otimes v) \Phi(a)-\left(\mu \otimes v^{\prime}\right) \Phi(a)\right| \neq 0 .
$$

By the definition of $d$, we have $d\left(v, v^{\prime}\right) \geq \rho_{L}\left((\mu \otimes v) \Phi,\left(\mu \otimes v^{\prime}\right) \Phi\right)$. On the other hand, by the definition of $\rho_{L}$,

$$
\begin{aligned}
\rho_{L}\left((\mu \otimes v) \Phi,\left(\mu \otimes v^{\prime}\right) \Phi\right) & \geq\left|(\mu \otimes v) \Phi\left(\frac{a}{L(a)}\right)-\left(\mu \otimes v^{\prime}\right) \Phi\left(\frac{a}{L(a)}\right)\right| \\
& =\frac{\left|(\mu \otimes v) \Phi(a)-\left(\mu \otimes v^{\prime}\right) \Phi(a)\right|}{L(a)} .
\end{aligned}
$$

Thus, (3.1) is satisfied and $L_{d}(c) \leq L(a)$.

Now, we show that the map $v \longmapsto v(c)$ on $S(\mathfrak{C})$ is continuous with $\tau_{d}$. Let $v_{n} \rightarrow v$ be a convergent sequence in $S(\mathfrak{C})$ with the metric $d$. Thus, by the definition of $d$, we have

$$
\rho_{L}\left(\left(\mu \otimes v_{n}\right) \Phi,(\mu \otimes v) \Phi\right) \rightarrow 0 .
$$

Therefore, by Proposition 2.4,

$$
v_{n}(c)=\left(\mu \otimes v_{n}\right) \Phi(a) \rightarrow(\mu \otimes v) \Phi(a)=v(c) .
$$

Proposition 3.5. With the above assumptions, suppose that $(\mathfrak{A}, \mathscr{A}, L)$ is a $Q M$ space and the linear span of

$$
G=\left\{\left(\mu \otimes i d_{\mathfrak{C}}\right) \Phi(a): \quad \mu \in S(\mathfrak{B}), a \in \mathfrak{A}\right\}
$$

is dense in $\mathfrak{C}$ (for example $\Phi$ is surjective). Then $\left(\mathfrak{C}, \mathscr{C}, L_{d}\right)$ is a $Q M$ space.

Proof. Since $\mathscr{A}$ is dense in $\mathfrak{A}$ and the linear span of $G$ is dense in $\mathfrak{C}$, we have

$$
G_{0}=\left\{\left(\mu \otimes i d_{\mathfrak{C}}\right) \Phi(a): \quad \mu \in S(\mathfrak{B}), a \in \mathscr{A}\right\}
$$

is dense in $\mathfrak{C}$. On the other hand, by Lemma $3.4, G_{0} \subset \mathscr{C}$. Thus $\mathscr{C}$ is dense in $\mathfrak{C}$ and $\left(\mathfrak{C}, \mathscr{C}, L_{d}\right)$ is a QM space.

Example 3.6. Let $\mathfrak{A}$ and $\mathfrak{C}$ be unital $C^{*}$-algebras. Suppose that $\mathfrak{A} \otimes \mathfrak{C}$ has a $Q S M$ structure. Consider *-homomorphisms

$$
i d: \mathfrak{A} \otimes \mathfrak{C} \rightarrow \mathfrak{A} \otimes \mathfrak{C} \quad \text { and } \quad F: \mathfrak{A} \otimes \mathfrak{C} \rightarrow \mathfrak{C} \otimes \mathfrak{A},
$$

where $F$ is the flip map, i.e. $F(a \otimes c)=c \otimes a$ for $a \in \mathfrak{A}, c \in \mathfrak{C}$. Then

$$
\left(\mathfrak{C}, i d_{\mathfrak{A} \otimes \mathfrak{C}}\right) \quad \text { and } \quad(\mathfrak{A}, F)
$$

are quantum families of morphisms. Thus $\mathfrak{A}$ and $\mathfrak{C}$ have naturally QSM structures. Also, by Proposition 3.5, if $\mathfrak{A} \otimes \mathfrak{C}$ has a QM structure then so are $\mathfrak{A}$ and $\mathfrak{C}$.

Example 3.7. Let $\mathfrak{A}$ be a unital $C^{*}$-algebra and suppose that $\mathfrak{A}$ has a $Q S M$ structure. Let $\Phi: \mathfrak{A} \rightarrow \mathfrak{B}$ be a unital $*$-homomorphism. Then $(\mathfrak{B}, \Phi)$ can be considered as a quantum family of morphisms from $\mathfrak{A}$ to $\mathbb{C}$. Thus $\mathfrak{B}$ naturally has a $Q S M$ structure. Also, if $\Phi$ is surjective and $\mathfrak{A}$ has a $Q M$ structure, then by Proposition $3.5, \mathfrak{B}$ has a QM structure.

\section{The commutative case}

In this last section we study induced metric structures on ordinary families of maps.

Lemma 4.1. Let $(X, \tau)$ be a compact Hausdorff space and let $d$ be a pseudo-metric on $S(\mathbf{C}(X, \tau))$ such that $\tau_{d}$ is weaker than the $w^{*}$ topology. Let $\mathscr{C}$ be the space of all $c \in \mathbf{C}(X, \tau)$ such that the map $v \longmapsto v(c)$ is continuous on $S(\mathbf{C}(X, \tau))$ and $L_{d}(c)<\infty$. Consider the semi norm $L_{d}: \mathscr{C} \rightarrow[0,+\infty)$. Then for every $x, x^{\prime} \in X, d\left(\delta_{x}, \delta_{x^{\prime}}\right)=\rho_{L_{d}}\left(\delta_{x}, \delta_{x^{\prime}}\right)$.

(We remark that Lemma 4.1 is different from part i) of Proposition 2.2.)

Proof. Let $x, x^{\prime}$ be in $X$. By the definition of $\rho_{L_{d}}$, we have

$$
\rho_{L_{d}}\left(\delta_{x}, \delta_{x^{\prime}}\right)=\sup \left\{\left|a(x)-a\left(x^{\prime}\right)\right|: \quad a \in \mathscr{C}, L_{d}(a) \leq 1\right\} .
$$

Let $a \in \mathscr{C}$ and $L_{d}(a) \leq 1$. If $d\left(\delta_{x}, \delta_{x^{\prime}}\right)=0$, then $a(x)=a\left(x^{\prime}\right)$ since the map $\delta_{x} \longmapsto \delta_{x}(a)=a(x)$ is continuous with $d$, thus (4.1) implies that

$$
\rho_{L_{d}}\left(\delta_{x}, \delta_{x^{\prime}}\right)=d\left(\delta_{x}, \delta_{x^{\prime}}\right)=0 .
$$

Now, suppose that $d\left(\delta_{x}, \delta_{x^{\prime}}\right) \neq 0$. Since $1=L_{d}(a) \geq \frac{\left|a(x)-a\left(x^{\prime}\right)\right|}{d\left(\delta_{x}, \delta_{x^{\prime}}\right)}$, we have $d\left(\delta_{x}, \delta_{x^{\prime}}\right) \geq\left|a(x)-a\left(x^{\prime}\right)\right|$, thus (4.1) implies that $\rho_{L_{d}}\left(\delta_{x}, \delta_{x^{\prime}}\right) \leq$ $d\left(\delta_{x}, \delta_{x^{\prime}}\right)$. Now, define a map $b_{x}$ on $X$ by $b_{x}(y)=d\left(\delta_{x}, \delta_{y}\right)$. Then $b_{x} \in \mathscr{C}$ and $L_{d}\left(b_{x}\right) \leq 1$. Thus

$$
\rho_{L_{d}}\left(\delta_{x}, \delta_{x^{\prime}}\right) \geq\left|b_{x}(x)-b_{x}\left(x^{\prime}\right)\right|=d\left(\delta_{x}, \delta_{x^{\prime}}\right) .
$$

This completes the proof. 
Theorem 4.2. Let $(X, \tau),\left(Y, \tau^{\prime}\right),\left(Z, \tau^{\prime \prime}\right)$ be compact Hausdorff spaces and let $d_{0}$ be a pseudo-metric on $X$ such that $\tau_{d_{0}} \subset \tau$. Let

$$
F: Y \times Z \rightarrow X
$$

be a continuous map with $\tau, \tau^{\prime}, \tau^{\prime \prime}$, and define a pseudo-metric $d_{1}$ on $Z$ by

$$
d_{1}\left(z, z^{\prime}\right)=\sup _{y \in Y} d_{0}\left(F(y, z), F\left(y, z^{\prime}\right)\right) .
$$

With the canonical identification $\mathbf{C}\left(Y \times Z, \tau^{\prime} \times \tau^{\prime \prime}\right) \cong \mathbf{C}\left(Y, \tau^{\prime}\right) \otimes \mathbf{C}\left(Z, \tau^{\prime \prime}\right)$ let

$$
\hat{F}: \mathbf{C}(X, \tau) \rightarrow \mathbf{C}\left(Y, \tau^{\prime}\right) \otimes \mathbf{C}\left(Z, \tau^{\prime \prime}\right)
$$

be defined by $\hat{F}(a)=a F$, for $a \in \mathbf{C}(X, \tau)$. Let

$$
\left(\mathbf{C}\left(Z, \tau^{\prime \prime}\right), \mathscr{C}, N\right)
$$

be the QSM space induced by $Q S M$ space $\left(\mathbf{C}(X, \tau), \mathbf{L i p}\left(X, d_{0}\right),\|\cdot\|_{d_{0}}\right)$ and quantum family of morphisms $\left(\mathbf{C}\left(Z, \tau^{\prime \prime}\right), \hat{F}\right)$. Then the following are satisfied.

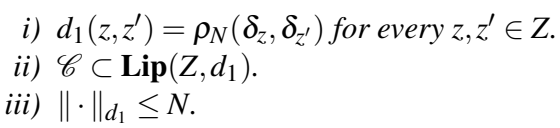

Proof. i) Let $L=\|\cdot\|_{d_{0}}$. Let us recall the definition of $\left(\mathbf{C}\left(Z, \tau^{\prime \prime}\right), \mathscr{C}, N\right)$. Let $d$ be the pseudo-metric on $S\left(\mathbf{C}\left(Z, \tau^{\prime \prime}\right)\right)$ defined by

$$
d\left(v, v^{\prime}\right)=\sup \left\{\rho_{L}\left((\mu \otimes v) \hat{F},\left(\mu \otimes v^{\prime}\right) \hat{F}\right): \quad \mu \in S\left(\mathbf{C}\left(Y, \tau^{\prime}\right)\right)\right\} .
$$

Then $N=L_{d}$ and $\mathscr{C}$ is the space of all $c \in \mathbf{C}\left(Z, \tau^{\prime \prime}\right)$ such that the map $v \longmapsto v(c)$ on $S\left(\mathbf{C}\left(Z, \tau^{\prime \prime}\right)\right)$ is continuous with $d$ and $N(c)<\infty$. By Lemma 4.1, we have,

$$
d\left(\delta_{z}, \delta_{z^{\prime}}\right)=\rho_{N}\left(\delta_{z}, \delta_{z^{\prime}}\right),
$$

for every $z, z^{\prime} \in Z$. Now, we explain the relation between $d_{1}$ and $d$.

Let $z, z^{\prime} \in Z$ and $y \in Y$. Then

$$
\left(\delta_{y} \otimes \delta_{z}\right) \hat{F}=\delta_{F(y, z)} \quad \text { and } \quad\left(\delta_{y} \otimes \delta_{z^{\prime}}\right) \hat{F}=\delta_{F\left(y, z^{\prime}\right)} .
$$

On the other hand, by Proposition 2.2, for every $x, x^{\prime} \in X, d_{0}\left(x, x^{\prime}\right)=\rho_{L}\left(\delta_{x}, \delta_{x^{\prime}}\right)$. Thus

$$
\rho_{L}\left(\left(\delta_{y} \otimes \delta_{z}\right) \hat{F},\left(\delta_{y} \otimes \delta_{z^{\prime}}\right) \hat{F}\right)=d_{0}\left(F(y, z), F\left(y, z^{\prime}\right)\right) .
$$

This formula together with the definitions of $d$ and $d_{1}$, show that

$$
d_{1}\left(z, z^{\prime}\right) \leq d\left(\delta_{z}, \delta_{z^{\prime}}\right) .
$$

Let $\mu \in S\left(\mathbf{C}\left(Y, \tau^{\prime}\right)\right)$ be arbitrary. We consider $\mu$ as a probability Borel regular measure on $\left(Y, \tau^{\prime}\right)$. Then for every $a \in \mathbf{L i p}\left(X, d_{0}\right)$ with $\|a\|_{d_{0}} \leq 1$, we have,

$$
\begin{aligned}
\left|\left(\mu \otimes \delta_{z}\right) \hat{F}(a)-\left(\mu \otimes \delta_{z^{\prime}}\right) \hat{F}(a)\right| & =\left|\int_{Y}\left(a F(y, z)-a F\left(y, z^{\prime}\right)\right) d_{\mu}(y)\right| \\
& \leq \int_{Y}\left|a(F(y, z))-a\left(F\left(y, z^{\prime}\right)\right)\right| d_{\mu}(y) .
\end{aligned}
$$

For every $y \in Y$, by Lemma 2.1,

$$
\left|a(F(y, z))-a\left(F\left(y, z^{\prime}\right)\right)\right| \leq d_{0}\left(F(y, z), F\left(y, z^{\prime}\right)\right) .
$$

Therefore, we have

$$
\left|a(F(y, z))-a\left(F\left(y, z^{\prime}\right)\right)\right| \leq d_{1}\left(z, z^{\prime}\right) .
$$

(4.5) and (4.4) implies that

$$
\left|\left(\mu \otimes \delta_{z}\right) \hat{F}(a)-\left(\mu \otimes \delta_{z^{\prime}}\right) \hat{F}(a)\right| \leq d_{1}\left(z, z^{\prime}\right) .
$$

Therefore, by the definition of $d$,

$$
d\left(\delta_{z}, \delta_{z^{\prime}}\right) \leq d_{1}\left(z, z^{\prime}\right) .
$$

Now, by (4.6) and (4.3), $d\left(\delta_{z}, \delta_{z^{\prime}}\right)=d_{1}\left(z, z^{\prime}\right)$, and thus by (4.2),

$$
d_{1}\left(\delta_{z}, \delta_{z^{\prime}}\right)=\rho_{N}\left(\delta_{z}, \delta_{z^{\prime}}\right)
$$

for every $z, z^{\prime} \in Z$, and i) is satisfied. ii) and iii) are immediate consequence of i) and definitions of $\mathscr{C},\|\cdot\|_{d_{1}}$ and $N$. 


\section{Conclusion}

In this note, we introduced the new concept of compact quantum pseudo-metric space as a generalization of the concept of compact quantum metric space. The $\mathrm{C}^{*}$-algebraic examples of the latter concept, which has been introduced by Rieffel, are very restricted. But, by using the concept of quantum family of maps, it was denoted that the source of examples for ( $\mathrm{C}^{*}$-algebraic) quantum pseudo-metric spaces are very wider than those for $\left(\mathrm{C}^{*}\right.$-algebraic) quantum metric spaces.

\section{References}

[1] A. Connes, Compact metric spaces, Fredholm modules and hyperfiniteness, Ergo. Th. Dyn. Sys. 9 (1989), $207-220$.

[2] A. Connes, Noncommutative Geometry, Academic Press, 1994

[3] G. Kuperberg, N. Weaver, A von Neumann algebra approach to quantum metrics/quantum relations, Vol. 215, no. 1010. American Mathematical Society, 2012.

[4] S. T. Rachev, Probability Metrics and the Stability of Stochastic Models, John Wiley and Sons, 1991.

[5] M. A. Rieffel, Metrics on states from actions of compact groups, Doc. Math. 3 (1998), 215-229.

[6] M. A. Rieffel, Metrics on state spaces, Doc. Math. 4 (1999), 559-600.

[7] M. A. Rieffel, Gromov-Hausdorff distance for quantum metric spaces, Mem. Amer. Math. Soc. 168 (2004), 1-65.

[8] M. A. Rieffel, Compact quantum metric spaces, Contemp. Math. 365 (2004), 315-330.

[9] M. A. Rieffel, Leibniz seminorms for Matrix algebras converge to the sphere, Quanta of maths, Clay Math. Proc., vol. 11, Amer. Math. Soc., Providence, RI, 2010, 543-578.

[10] M. M. Sadr, Quantum functor Mor, Math. Pannonica 21 no. 1 (2010), 77-88

[11] M. M. Sadr, A kind of compact quantum semigroups, Int. J. Math. Math. Sci. 2012 (2012), Article ID 725270,10 pages.

[12] M. M. Sadr, On the quantum groups and semigroups of maps between noncommutative spaces, Czechoslovak Math. J. 67 no. 1 (2017), $97-121$.

[13] M. M. Sadr, Ouantum metrics on noncommutative spaces, available at https://arxiv.org/pdf/1606.00661.pdf

[14] M. M. Sadr, Metric operator fields, available at https://arxiv.org/pdf/1705.03378.pdf

[15] P. M. Sołtan, Quantum families of maps and quantum semigroups on finite quantum spaces, J. Geom. Phys. 59 (2009), $354-368$.

[16] S. L. Woronowicz, Pseudogroups, pseudospaces and Pontryagin duality, Proceedings of the International Conference on Mathematical Physics, Lausanne 1979 , Lecture Notes in Physics 116, 407-412. 